\title{
Environnement capacitant et engagement des opérateurs : une mise en débat à partir de l'activité des techniciens de la base polaire Dumont D'Urville
}

Enabling environment and operator engagement: a debate based on the activity of technicians at the Dumont D'Urville polar station

\section{Aude Villemain et Yannick Lémonie}

\section{OpenEdition}

\section{Journals}

Édition électronique

URL : http://journals.openedition.org/activites/1063

DOI : 10.4000/activites.1063

ISSN : 1765-2723

Éditeur

ARPACT - Association Recherches et Pratiques sur les ACTivités

\section{Référence électronique}

Aude Villemain et Yannick Lémonie, «Environnement capacitant et engagement des opérateurs : une mise en débat à partir de l'activité des techniciens de la base polaire Dumont D'Urville ", Activités [En ligne], 11-2 | Octobre 2014, mis en ligne le 15 octobre 2014, consulté le 19 avril 2019. URL : http:// journals.openedition.org/activites/1063; DOI : 10.4000/activites.1063

\section{(c) (i) $\odot$}

Activités est mis à disposition selon les termes de la licence Creative Commons Attribution - Pas d'Utilisation Commerciale - Pas de Modification 4.0 International. 


\title{
Environnement capacitant et engagement des opérateurs : une mise en débat à partir de l'activité des techniciens de la base polaire Dumont D'Urville
}

\author{
Aude Villemain \\ Université de Reims Champagne-Ardenne, Centre de Recherche sur le Travail et le Développement (CRTD) \\ Conservatoire national des arts et métiers (Cnam) \\ 41, rue Gay-Lussac, 75005 Paris \\ aude.villemain@univ-reims.fr
}

Yannick Lémonie

Centre de Recherche sur le Travail et le Développement (CRTD)

Conservatoire national des arts et métiers (Cnam)

41, rue Gay-Lussac, 75005 Paris

\begin{abstract}
Enabling environment and operator engagement: a debate based on the activity of technicians at the Dumont D'Urville polar station. Learning in the workplace is a serious issue in ergonomics. In this perspective, constructive ergonomics (Falzon, 2013) proposes the enabling environment concept to establish a model of work environment characteristics, leading to learning and to development in and through work. The purpose of the present study is to examine the learning and development of wintering operators within two specific models, in order to highlight learning conditions at work: the enabling environment and Workplace Learning (Billett, 2001) approaches. This article proposes a work study based on nine wintering technicians isolated for twelve months in the French Dumont D'Urville station in Antarctica,. The main results show that polar constraints, specific work organisation involving autonomy, and the resources available to technicians enable them to acquire and develop new skills. In this perspective, the article discusses and proposes a definition of environment characteristics which enable learning in and through work.
\end{abstract}

KEY-WORDS

learning, productive activity, enabling environment, polar conditions, engagement

\section{Introduction}

La question de l'apprentissage et du développement au travail est devenue un sujet d'importance dans divers champs disciplinaires. Ainsi, l'analyse de l'activité, tant en ergonomie de langue française que dans de nombreux travaux récents de sciences de l'éducation, s'intéresse aux conditions, qui en situation de travail, sont sources de développement et d'apprentissage. Concevoir des situations de travail participant au développement professionnel est ainsi devenu un enjeu majeur et essentiel de l'ergonomie (Falzon, 2013).

Le programme de recherche ouvert par l'ergonomie constructive (Falzon, 2013 ; Falzon \& Mollo, 2009) s'inscrit dans cette perspective développementale, en structurant ses apports autour du concept d'environnement capacitant (Pavageau, Nascimento, \& Falzon, 2007). De fait, dans cette conception de l'ergonomie, l'apprentissage et le développement des opérateurs en situation de travail deviennent centraux dans l'analyse, et ce, de manière 
concomitante et complémentaire aux autres niveaux d'action de l'ergonome, notamment en termes de réduction des contraintes délétères pour la santé des opérateurs, mais également en termes d'adaptation à l'hétérogénéité des acteurs. Autrement dit, si l'objectif de l'ergonomie constructive est le développement, celle-ci vise à concevoir des environnements de travail permettant aux opérateurs de «développer de nouveaux savoirs et de nouvelles connaissances, d'élargir leurs possibilités d'action » (Falzon, 2013, p. 4).

Le développement de ce programme de recherche et la définition de tels environnements capacitants impliquent l'analyse et la modélisation des contraintes et des ressources, qui permettent ou qui entravent l'apprentissage et le développement en situation de travail. À ce titre, les travaux en ergonomie ont sans doute à gagner à être confrontés à ceux relevant des sciences de l'éducation. Dans ce domaine, le courant anglo-saxon du Workplace Learning (e.g. Billett, 1992; Billett, Johnson, Thomas, Sim, Hay, \& Ryan, 2012) offre une modélisation et des perspectives qu'il s'agit de discuter au regard de la notion d'environnement capacitant telle qu'elle a pu être développée et définie dans les différents travaux d'ergonomie constructive.

L'objet de cet article consiste ainsi en une mise en discussion critique de ces deux modélisations (environnement capacitant et Workplace Learning) traitant de l'apprentissage et du développement en situation de travail à partir des données issues d'une recherche menée sur la base de Dumont D’Urville (DDU) en Antarctique.

Les caractéristiques particulières de ce contexte de travail en font une situation d'étude privilégiée (Grison \& Riff, 2002), sur un terrain de recherche qui n'a, à notre connaissance, jamais été investigué dans le cadre d'une intervention ergonomique. D'une part, les opérateurs qui viennent travailler sur la base DDU ne sont affectés que pour une durée limitée (un an), d'autre part les conditions géographiques et climatiques (isolement, froid, durée limitée du jour, matériels et matériaux particuliers, etc.) impliquent une adaptation importante aussi bien du contenu que des conditions de réalisation du travail. L'environnement polaire constitue un environnement extrême au sens où l'entendent Wolff et Sperandio (2004, p. 115). Si les auteurs notent que le travail en condition extrême est «un défi que l'ergonomie n'a pas pour ambition de relever» du fait de son caractère «en limite », c'est justement ce caractère limite qui invite à s'y intéresser ici : il est vecteur d'apprentissage et de développement des savoir-faire de métier et de prudence (Cru, 1995). Contre une vision négative et essentiellement contraignante des environnements extrêmes, nous pensons ainsi la base DDU comme un terrain particulièrement propice pour étudier l'apprentissage et le développement des opérateurs au travail et pour une mise en discussion critique des modélisations qui en font leur objet.

\section{1.- Les modélisations des conditions entravant ou permettant le développement et l'apprentissage}

\section{1.- L'approche par les capabilités et la notion d'environnement capacitant}

L'ergonomie constructive part du constat que l'ergonomie ne peut pas se satisfaire d'une conception statique du travail qui conduirait à définir ou à concevoir des systèmes de travail certes adaptés aux opérateurs, mais figés et potentiellement contraignant dès lors que les opérateurs eux-mêmes évoluent. En ce sens, «l'objectif de l'ergonomie doit être le développement » (Falzon, 2013, p. 1).

Cette orientation développementale de l'ergonomie constructive s'appuie sur les travaux de Sen $(1993,2000,2012)$ et principalement sur le concept de «capabilité ». Les capabilités renvoient à l'ensemble des fonctionnements possibles pour un individu et donc à un champ des possibles et à la latitude effective dont dispose un individu. Les capabilités impliquent 
ainsi des capacités, mais ne s'y réduisent cependant pas (Falzon \& Mollo, 2009). Si les capacités relèvent du savoir-faire, les capabilités renvoient à la possibilité effective de mise en œuvre de ces capacités. Ainsi la mise en œuvre d'une capacité ne dépend pas seulement de la disponibilité de cette capacité chez l'individu, mais d'un ensemble de conditions organisationnelles, techniques, sociales..., qui s'actualisent et se transforment par la suite en capabilités dans une situation donnée (Zimmerman, 2008). Ces conditions organisationnelles, techniques et sociales renvoient à des facteurs de conversion permettant de convertir des capacités en possibilités réelles (Falzon, 2013). Dans une analyse du développement des compétences en situation de travail, Delgoulet et Vidal-Gomel (2013) identifient les conditions favorables et défavorables au développement de compétences. En ce qui concerne les conditions favorables (les facteurs de conversion), les auteures identifient comme première, à la suite des travaux de Vygotski (1934/1997), la «zone proche de développement des individus ", c'est-à-dire le rapport entre ce que les opérateurs sont en mesure de faire (les capacités) et ce qu'ils peuvent faire (capabilités) grâce à la médiation par les pairs ou par des outils d'aide présents comme ressources au sein des situations de travail (les facteurs de conversion). Parmi les conditions défavorables au développement de compétences, les auteures identifient des prescriptions et des contraintes temporelles trop fortes, ou à l'inverse, une absence totale de prescriptions générant de l'incertitude.

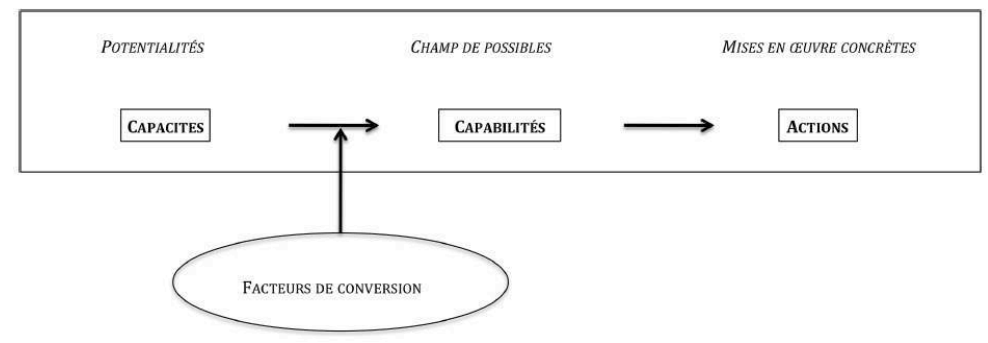

Figure 1 : Relations entre capacités, capabilités, facteurs de conversion et actions

Figure 1: Relationships between capacities, capabilities, conversion factors and actions

Cette approche par les capabilités permet d'envisager la conception d'environnements capacitants définis autour de trois caractéristiques essentielles (Falzon, $2005 ; 2006$ ) :

— D'un point de vue préventif, c'est un environnement permettant de préserver les capacités futures d'action ;

- D'un point de vue universel, c'est un environnement prenant en compte des différences interindividuelles (sexe, âge, culture), qui compense les déficiences individuelles (vieillissement, maladie, incapacités). Ce type d'environnement prévient donc l'exclusion et favorise l'intégration, l'inclusion et la reconnaissance sociale.

- D'un point de vue développemental, c'est un environnement permettant le développement de nouvelles compétences et de nouveaux savoirs, l'élargissement des possibilités d'action et du degré de contrôle sur la tâche et sur l'activité.

De ces trois caractéristiques qui relèvent de trois niveaux d'action de l'ergonome, le troisième représente un apport essentiel de l'ergonomie constructive qui justifie que l'on se centre plus particulièrement sur cette dimension dans le cadre de cet article. En effet, l'ergonomie constructive consiste à souligner que l'élargissement des possibilités d'action de l'opérateur, son apprentissage et son développement sont susceptibles de permettre à la fois une meilleure efficacité et de construire sa santé au travail. L'analyse et la modélisation des conditions favorables aux apprentissages dans les situations de travail, donc des facteurs de conversion, deviennent en ce sens un enjeu majeur pour le développement de l'ergonomie constructive et la définition de tels environnements capacitants. 


\section{2.- Apprendre sur le lieu de travail : Workplace Learning}

La question de l'apprentissage au travail est au cœur du courant du Workplace Learning (Bourgeois \& Durand, 2012). En considérant que les sciences de l'éducation se sont principalement centrées sur les institutions éducatives et ont privilégié l'étude de l'apprentissage dans des situations scolaires ou de formation (Billett, 2002), les chercheurs de ce courant ont tenté de comprendre comment l'apprentissage prenait place dans le cadre du travail, à partir de théories générales sur l'apprentissage d'inspiration socio-historique (Billett, 2009). Les études ont ainsi articulé une double dimension, épistémique et transformative : répondre à la question des caractéristiques des environnements de travail qui influent sur l'apprentissage, c'est s'intéresser à rendre les environnements de travail plus formatifs. En effet, pour Billett (2002, p. 458) «the procedural goals are associated with the capacity to provide experiences to develop and sustain vocational practice in workplace settings through work and throughout working lives ».

Pour Billett $(2001,2009)$ deux types de facteurs influencent les apprentissages en situation de travail (Figure 2) :

— des facteurs liés à l'environnement de travail, qui peuvent soit entraver, soit favoriser l'apprentissage au travail, sont conceptualisés en termes «d'affordance ». Il est à noter ici que l'usage du concept d'affordance renvoie à une signification quelque peu différente de chez Gibson (1977) où celle-ci est liée à la configuration de l'action sur la base d'indices essentiellement visuels. Dans ce courant, la notion d'affordance renvoie à la variété des ressources offertes par l'environnement et susceptibles d'être prises en compte par l'opérateur pour apprendre et se développer sur le lieu de travail.

— des facteurs individuels conceptualisés en termes d'engagement des individus, qui représente la part agentive ${ }^{1}$ des opérateurs et traduit la signification qu'ils accordent aux affordances présentes dans leur environnement de travail. Pour Billett, Baker et HernonTinning (2004, p.237) «despite goal-directed activities and interactions and their distribution being shaped by social norms and practices, individuals exercise their agency in determining how they interpret and engage in social practice. Ultimately, this agency decides what they learn through their engagement $»$.
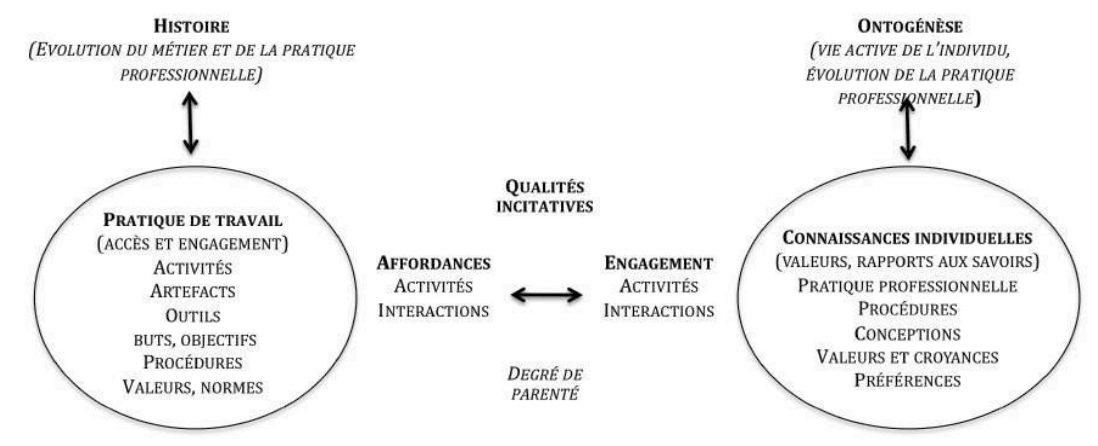

Figure 2: Affordances et engagement dans les apprentissages au travail (Billett, 2012)

Figure 2: Affordances and engagement in Workplace Learning (Billett, 2012)

D'inspiration socio-constructiviste, le courant Workplace Learning insiste largement sur les ressources sociales de l'environnement de travail. Le rôle des interactions sociales est à ce titre prépondérant. Ainsi, une des premières affordances est la possibilité d'être accompagné par un collègue plus expérimenté (Billett, 2001). Ceci constitue une ressource indispensable pour l'accès à des connaissances sans doute peu visibles dans l'environnement de travail. Par

\footnotetext{
${ }^{1}$ Sa capacité à agir sur les autres et sur l'environnement de travail.
} 
exemple, Billett (2002) montre bien, à partir d'une étude dans un salon de coiffure, que si les professionnels peuvent observer le processus de coloration des cheveux, cette seule observation ne leur donne pas accès aux connaissances sous-jacentes nécessaires à la maîtrise de ce savoir-faire. Dans ce cadre, le pair tuteur permet la transmission des connaissances nécessaires à l'apprentissage de la maîtrise d'un savoir-faire. Au-delà de cette dimension interactionnelle, les travaux de ce courant insistent également sur la dimension d'apprentissage inscrite dans des environnements a priori «muets». Ces environnements «parlent » en définitive : l'agencement où l'aménagement spatio-temporel, l'environnement matériel et technique sont susceptibles d'influer sur l'activité et l'apprentissage des opérateurs, et peuvent même être intentionnellement conçus à des fins d'apprentissage. Ainsi, les artefacts peuvent cristalliser un certain nombre de connaissances (Rabardel, 1995) qui guident efficacement l'action et l'apprentissage au travail. A contrario, certains environnements de travail peuvent limiter les opportunités d'apprentissage : «issues of learning within work intensive environments, hectic, differentiated, dynamic and unpredictable work circumstances, and securing access to knowledge that is not readily accessible through workplace situations are just some circumstances of contemporary work that can limit opportunities of learning » (Billett, 2012, p. 128). La notion d'affordance renvoie donc à la variété des ressources susceptibles d'être offertes par l'environnement pour apprendre et se développer sur le lieu de travail: elles peuvent être conceptualisées en termes de potentialités.

Toutefois, si l'environnement de travail offre des ressources, c'est le degré d'engagement des opérateurs qui conditionne réellement les apprentissages. Ce sont les facteurs individuels qui font que les opérateurs usent ou au contraire rejettent les affordances de l'environnement de travail : "ainsi, la participation peut prendre la forme d'un rejet de ces affordances, d'un usage actif, voire de tentative de les dépasser ou de les contourner» (Billett, 2009, p. 42). Plusieurs facteurs individuels sont susceptibles de moduler le degré d'engagement que les opérateurs consentent dans leur activité : leurs priorités, ou leur centre d'intérêt (Glassman, 2001), les valeurs et les croyances des individus, etc. Ainsi, le degré d'engagement des individus dans les situations de travail est lié en grande partie à leur trajectoire biographique. Dans ce cadre, c'est moins les affordances qui conditionnent le degré d'engagement que la perception que les opérateurs en ont. Ainsi, Billett (2009) modélise l'apprentissage au travail comme la conséquence du degré de parenté entre les affordances de l'environnement de travail et l'engagement de l'individu. Au plan méthodologique, cette conceptualisation de l'apprentissage et du développement au travail implique inévitablement une prise en compte non seulement des affordances et des fondements de son engagement, mais également des négociations entre ces dualités.

\section{3.- Tension entre modèles : une mise en question}

Bien que ne mobilisant pas les mêmes concepts, les deux approches peuvent apparaître relativement voisines l'une de l'autre et complémentaires sur bien des points. En premier lieu les deux modélisations insistent sur le caractère potentiellement formatif des environnements de travail. En second lieu, toutes deux visent à spécifier les conditions qui dans ces environnements permettent ou entravent l'apprentissage et le développement au travail. Ainsi, les notions d'affordance et de facteurs de conversion apparaissent relativement proches quant à leur usage. Nous avons en effet souligné comment les facteurs de conversion pouvaient être rapprochés des conditions qui favorisent l'apprentissage-développement au travail : la zone proximale de développement, la médiation par les pairs, par la situation, le temps suffisant, etc. De la même manière, la notion d'affordance en est proche dans la mesure où elle renvoie également à la médiation par les pairs ou par les situations de travail.

Au-delà de ces similitudes, il s'agit de mettre en tension les deux approches pour faire apparaître les questions empiriques permettant de développer le modèle des environnements capacitants en le mettant à l'épreuve des faits. 
L'approche par les capabilités, en prenant appui sur une théorie de la liberté, ne vise pas en tant que tel à modéliser ou à comprendre les raisons qui font que les opérateurs s'emparent ou au contraire rejettent les ressources que les environnements leur offrent. Elle permet de mettre l'accent sur les conditions nécessaires pour la mise en œuvre réelle des capacités dans les situations de travail sans toutefois spécifier les usages qui sont faits de ces conditions. Pour donner un exemple, la présence d'un pair expert et d'une organisation permettant l'échange peut être entendue comme un facteur de conversion favorisant l'apprentissagedéveloppement au travail. Formulée comme cela, la modélisation ne met pas l'accent sur la manière dont les opérateurs s'emparent de ces conditions. Or, pour des conditions similaires, les usages et les apprentissages qui en découlent peuvent être très différents d'un opérateur à un autre. Au contraire, l'approche du Workplace Learning modélise ce point en spécifiant que le rejet ou l'usage des affordances est lié à la congruence entre l'engagement des opérateurs et les affordances de l'environnement de travail. La notion d'engagement apparaît ici essentielle pour saisir la manière dont les opérateurs s'emparent des affordances. Toutefois, dans un environnement de travail, il n'existe jamais une seule affordance ou un unique facteur de conversion. La multiplicité des ressources potentielles qu'offre l'environnement de travail pose la question de l'usage que font les opérateurs de ces ressources et de leur hiérarchie effective dans leur activité de travail.

Nous touchons là à une deuxième différence essentielle de ces deux approches. Celle-ci tient au caractère intentionnel des apprentissages qui se développent dans la conception des environnements de travail. Si l'on suit la distinction opérée en didactique professionnelle entre activité productive et activité constructive sur le lieu de travail (Samurçay \& Rabardel, 2004), l'activité constructive constitue un effet de la dimension productive de l'activité. Dans le cadre de l'approche Workplace Learning c'est essentiellement la dimension constructive qui est valorisée. La dimension productive n'est guère prise en compte, l'essentiel étant que l'individu apprenne. Ceci permet de souligner un point critique des travaux de ce courant. En s'inspirant de théories relativement générales de l'apprentissage, la question des contenus est évacuée. Or, ce que les opérateurs apprennent sur le lieu de travail, c'est le travail lui-même. Il s'agit bien de comprendre comment les opérateurs mobilisent les ressources de l'environnement pour accomplir une activité productive par le biais de laquelle ils vont apprendre.

Une troisième différence entre ces deux approches concerne le rôle des contraintes qu'exerce la situation de travail sur les opérateurs. Dans l'approche Workplace Learning, l'accent est mis sur les ressources de l'environnement de travail qui favorisent l'apprentissage. Dans ce cas, les contraintes ne sont en aucun cas conçues comme potentiellement susceptibles d'affecter positivement les apprentissages. Or les contraintes ne sont peut-être pas à considérer comme obligatoirement négatives, comme le préconise l'approche d'ergonomie constructive. Celles liées à l'activité productive sont susceptibles, jusqu'à un certain seuil, d'impacter positivement l'apprentissage, comme par exemple l'augmentation de la charge de travail qui permettrait de développer de nouvelles stratégies chez les opérateurs. Dans l'approche d'ergonomie constructive, le travail d'un ergonome n'est pas d'éliminer toutes contraintes, mais bien de comprendre le rôle possible que celles-ci peuvent jouer dans l'apprentissage et le développement au travail. La question est dans ce cadre de déterminer quelles sont les contraintes acceptables et comment elles conditionnent l'apprentissage sur le lieu de travail.

La tension entre les deux modèles fait donc apparaitre ces trois questions qu'il s'agit de documenter sur le terrain particulier de la base Dumont D'Urville, en Antarctique. L'environnement polaire, considéré comme hostile du fait des températures basses, mais aussi de l'isolement, rendent en effet nécessaires l'ajustement et le développement des savoir-faire acquis en formation initiale par les opérateurs techniciens pour le maintien du fonctionnement de la base et la survie des personnels qui y habitent au cours de la période d'hivernage. La base DDU constitue à ce titre un terrain d'étude privilégié pour mener une 
discussion critique et comparative des travaux menés sur l'apprentissage-développement au travail autant dans le registre des environnements capacitants que dans celui du Workplace Learning.

\section{2.- Méthode}

\section{1.- Contexte}

Cette recherche a été menée en collaboration avec l'Institut Paul Émile Victor (IPEV), dans le cadre d'un programme déposé en 2010. Le recueil des données a été effectué sur la base française Dumont D'Urville en Terre Adélie en Antarctique en campagne d'été 2010-2011. Cette base offre un terrain scientifique riche, pour l'étude de l'ozone, de la glaciologie, de la sismologie ou encore de la biologie marine ou de l'ornithologie. 26 Français en moyenne hivernent entre 12 à 16 mois sur la base chaque année. Pour satisfaire les besoins des 11 scientifiques, 9 techniciens et 6 logisticiens assurent le bon fonctionnement de la base et donc la survie des hivernants.

Entre les mois de mars et octobre, les glaces se referment et rendent la base inaccessible pendant 8 mois. Le groupe de 26 hivernants est donc livré à lui-même, sans assistance possible, soumis aux contraintes climatiques (vent parfois de plus de $200 \mathrm{~km} / \mathrm{h}$, des températures allant jusqu'à $-40^{\circ} \mathrm{C}$ l'hiver, une nuit dominante entre les mois de juin et août). Cet environnement polaire contraint les individus à vivre en isolement (loin de tout et de tout le monde) et en confinement (sous le regard permanent des uns et des autres).

\section{2.- Population}

L'étude a été menée auprès de 9 techniciens hivernants masculins sortants d'hivernage (Moy. 34 ans ; E.T. 12,5 ans) appartenant à 9 corps de métiers techniques différents : un électricien, un plombier, un mécanicien de véhicules, un mécanicien de précision (conception de pièces), un chef central (production de l'énergie), un second central (assistant du chef central), un soudeur, un menuisier, un chef technique (responsable de tous les techniciens) ont participé à l'étude. Le tableau 1 récapitule les caractéristiques des techniciens en termes d'expérience professionnelle (allant de ceux qui sortent de l'école, soit moins d'un an d'expérience professionnelle, à plus de 10 ans d'expérience professionnelle) et d'hivernage (allant du premier hivernage au second).

\begin{tabular}{|c|c|c|}
\hline Activité professionnelle & Expérience professionnelle & Expérience d'hivernage \\
\hline Électricité (E) & $>10$ ans (marine) & $2^{\text {ème }}$ hivernage \\
Menuiserie (M) & $<1$ an & $1^{\text {er }}$ hivernage \\
Chaudronnier (C) & $<1$ an & $1^{\text {er }}$ hivernage \\
Responsable centrale (RC) & $>10$ ans & $2^{\text {ème }}$ hivernage \\
Assistant centrale (AC) & $<1$ an & $1^{\text {er }}$ hivernage \\
Plombier (P) & $>10$ ans & $1^{\text {er }}$ hivernage \\
Mécanicien de véhicule (MV) & $<5$ ans & $1^{\text {er }}$ hivernage \\
Mécanicien de précision (MP) & $<1$ an & $1^{\text {er }}$ hivernage \\
Responsable technique (RT) & $>10$ ans & $2^{\text {ème } h i v e r n a g e ~}$ \\
\hline
\end{tabular}

Tableau 1 : Caractéristiques des hivernants

Table 1: Characteristics of wintering personnel

\section{3.- Recueil de données}

Les données ont été recueillies par le premier auteur de cet article grâce à un travail en immersion d'environ 2 mois (décembre 2010, janvier 2011). Deux types de données ont été recueillis : 
(1) des observations ouvertes (et parfois participantes) de type ethnographique durant la réalisation du travail technique des hivernants. Nous avons observé pour chaque corps de métier une tâche effectuée en temps réel par le technicien sur son lieu de travail. Le choix de la tâche a été défini par les techniciens selon ce qui leur semblait le plus important à transmettre aux futurs hivernants lors de la relève de poste pendant l'été austral. Ces observations nous ont permis de préparer l'entretien suivant en comprenant le travail à réaliser, les moyens mis en œuvre pour le réaliser, afin d'orienter les questions vers la spécificité du métier. Lors de ces observations, les opérateurs étaient engagés dans la verbalisation (verbalisation à haute voix pendant leur activité de travail) de ce qu'il y avait à faire pour réaliser la tâche. Ces verbalisations ont été le support des entretiens semi-directifs;

(2) des entretiens semi-directifs (Moy : 1h) menés en deux temps de la manière suivante : (a) dans un premier temps il a été demandé à l'hivernant de parler de la réalisation de la tâche choisie dans le cadre de son activité professionnelle en France; (b) dans un deuxième temps il a été demandé aux hivernants de décrire leur activité lors de la réalisation de la même tâche sur la base Dumont D'Urville dans cet environnement durant les phases opératoires.

Chaque corps de métier a été observé sur une temporalité variable, en fonction des contraintes organisationnelles et climatiques sur la base, allant d'une journée complète de travail à trois jours.

Les entretiens ont tous été menés après l'observation des pratiques professionnelles. Pour des raisons de facilité d'intégration du protocole dans le quotidien de vie des hivernants, les entretiens ont été menés principalement sur leur lieu de travail.

\section{4.- Traitement des données}

Après transcriptions, les données d'entretiens, mais également les données d'observations participantes ont été analysées selon la théorie ancrée de Corbin \& Strauss (2008) par catégorisations inductives. Quatre grandes unités thématiques ont été relevées à l'issue de l'analyse de tous les verbatim : (1) les savoir-faire mobilisés lors de la réalisation de la tâche en France; (2) les contraintes de l'environnement polaire sur la réalisation du travail, déclinées en types de contraintes (isolement, confinement, climatique, et les incertitudes); (3) les nouveaux savoir-faire développés pour ajuster le travail aux contraintes polaires ; (4) les ressources utilisées pour résoudre les difficultés professionnelles rencontrées durant la réalisation de la tâche.

\section{3.- Résultats}

\section{1.- Développement de savoir-faire des techniciens face aux contraintes polaires}

Comme énoncé précédemment, l'environnement polaire renvoie à un environnement hostile reposant principalement sur quatre caractéristiques : l'isolement, le confinement, le climat et l'incertitude des activités à effectuer. Ces quatre aspects ont été formulés par les hivernants au cours des entretiens sous la forme de contraintes empêchant la réalisation du travail selon les modalités apprises au cours de leur formation ou utilisées lors de la pratique du métier en France. Le tableau 2 synthétise les contraintes énoncées dans chaque corps de métier ainsi que les adaptations à comprendre en termes de développement-apprentissage de nouveaux savoir-faire. 


\begin{tabular}{|c|c|c|c|}
\hline $\begin{array}{c}\text { Activités } \\
\text { professionnelles }\end{array}$ & Savoir-faire initial & $\begin{array}{l}\text { Impact des conditions } \\
\text { polaires sur le travail }\end{array}$ & $\begin{array}{l}\text { Développement de } \\
\text { nouveaux savoir-faire }\end{array}$ \\
\hline Menuisier & $\begin{array}{l}\text { Pose et dépose de } \\
\text { fenêtres et de portes }\end{array}$ & $\begin{array}{l}\text { Neige : infiltration par les } \\
\text { portes et les fenêtres qui ne } \\
\text { ferment plus } \\
\text { Vent }(200-250 \mathrm{~km} / \mathrm{h}): \\
\text { vibrations de bâtiment } \\
\text { provoquant l'ouverture des } \\
\text { portes et des fenêtres }\end{array}$ & $\begin{array}{l}\text { Savoir-faire de pose } \\
\text { spécifiques, avec des } \\
\text { matériaux spécifiques (portes } \\
\text { de chambre froide, avec des } \\
\text { poignées montées de porte à } \\
\text { l'envers et une ouverture vers } \\
\text { le haut) et pour organiser son } \\
\text { travail en fonction des } \\
\text { températures }\end{array}$ \\
\hline Chaudronnier & $\begin{array}{l}\text { Soudure selon } 3 \\
\text { techniques, dont celle au } \\
\text { gaz pour assembler des } \\
\text { grosses pièces (comme } \\
\text { des confections de } \\
\text { marches) }\end{array}$ & $\begin{array}{l}\text { Vent : soudure au gaz } \\
\text { impossible } \\
\text { Incertitude climatique }\end{array}$ & $\begin{array}{l}\text { Utilisation d'une autre } \\
\text { technique de soudure + } \\
\text { coûteuse en temps } \\
\text { Développement de savoir- } \\
\text { faire spécifiques à } \\
\text { l'organisation du travail en } \\
\text { fonction du vent et pour se } \\
\text { préserver du froid }\end{array}$ \\
\hline Électricien & $\begin{array}{l}\text { Utilisation de câbles qui } \\
\text { restent souples lors de } \\
\text { travaux extérieurs. } \\
\text { Aucune conséquence des } \\
\text { travaux extérieurs lors } \\
\text { du toucher d'outils. }\end{array}$ & $\begin{array}{l}\text { Froid : les matériaux } \\
\text { durcissent, les câbles cassent, } \\
\text { les joints de boîtiers électriques } \\
\text { durcissent (impossibilité de les } \\
\text { refermer); Les outils collent à } \\
\text { la peau ; La neige s'infiltre } \\
\text { dans les boîtiers électriques }\end{array}$ & $\begin{array}{l}\text { Utilisation de rubans adhésifs } \\
\text { spécifiques pour les joints } \\
\text { Utilisation de câbles } \\
\text { silicones ; Calculs de la } \\
\text { longueur de déroulement de } \\
\text { câble; } \\
\text { Développement de savoir- } \\
\text { faire spécifiques à } \\
\text { l'utilisation des câbles dans le } \\
\text { froid et pour se préserver du } \\
\text { froid }\end{array}$ \\
\hline $\begin{array}{l}\text { Mécanicien de } \\
\text { précision }\end{array}$ & $\begin{array}{l}\text { Utilisation d'une } \\
\text { diversité de machines } \\
\text { (meuleuse, fraiseuse) } \\
\text { permettant la confection } \\
\text { de pièces de toutes } \\
\text { formes. La meuleuse } \\
\text { facilite la confection de } \\
\text { pièces rondes. } \\
\text { Utilisation de machines } \\
\text { aux normes européennes } \\
\text { en concordance avec les } \\
\text { plans et les cotes }\end{array}$ & $\begin{array}{l}\text { Isolement : une seule grosse } \\
\text { machine mécanique pour } \\
\text { faciliter les réparations durant } \\
\text { l'hivernage (limité en arrivage } \\
\text { des pièces); } \\
\text { Confinement : place limitée sur } \\
\text { la base : choix d'une fraiseuse, } \\
\text { pas de meuleuse. Machines en } \\
\text { provenance d'Australie }\end{array}$ & $\begin{array}{l}\text { Utilisation de conversions } \\
\text { pour le lien entre les plans et } \\
\text { la normalisation de la } \\
\text { machine } \\
\text { Développement de savoir- } \\
\text { faire spécifiques pour faire } \\
\text { une pièce ronde avec une } \\
\text { fraiseuse }\end{array}$ \\
\hline $\begin{array}{l}\text { Mécanicien de } \\
\text { véhicule }\end{array}$ & $\begin{array}{l}\text { Formation } \\
\text { essentiellement en } \\
\text { électronique pour } \\
\text { réparer les véhicules }\end{array}$ & $\begin{array}{l}\text { Glace : engins à chenille } \\
\text { atypiques } \\
\text { Froid : problème de démarrage } \\
\text { et de boîte de vitesse } \\
\text { Neige : problème d'infiltration } \\
\text { de neige dans les liquides } \\
\text { Isolement }: \text { engins atypiques } \\
\text { datant de } 1960, \text { mécaniques } \\
\text { pour faciliter les réparations et } \\
\text { pour être autonomes dans les } \\
\text { réparations. Électronique } \\
\text { limitée. }\end{array}$ & $\begin{array}{l}\text { Développement d'un système } \\
\text { de fermeture contre les } \\
\text { infiltrations de neige } \\
\text { Création de panneaux } \\
\text { chauffants pour la boîte de } \\
\text { vitesse } \\
\text { Branchement des engins sur } \\
\text { secteur la nuit pour éviter que } \\
\text { les liquides (huile, eau) ne } \\
\text { gèlent } \\
\text { Développement de savoir- } \\
\text { faire spécifiques à ces types } \\
\text { d'engins confrontés à ces } \\
\text { températures et pour se } \\
\text { préserver du froid }\end{array}$ \\
\hline Chef centrale & $\begin{array}{l}\text { Gestion des systèmes de } \\
\text { production d'énergie et } \\
\text { d'eau par pompage }\end{array}$ & $\begin{array}{l}\text { Froid : l'eau pompée de l'océan } \\
\text { gèle sur le temps de sa } \\
\text { remontée au système. D'où } \\
\text { création d'un système plus } \\
\text { complexe et prototypique } \\
\text { permettant l'autonomie sur la } \\
\text { base : le bouilleur ; dispose de } \\
10 \text { mn pour remettre en route le } \\
\text { système en cas de black-out } \\
\text { avant que le système ne gèle } \\
\text { Isolement : a la responsabilité } \\
\text { de } 26 \text { vies humaines sur la base }\end{array}$ & $\begin{array}{l}\text { Utilisation d'un système } \\
\text { inconnu } \\
\text { Diagnostic et gestion des } \\
\text { pannes spécifiques } \\
\text { rapidement } \\
\text { Développement de savoir- } \\
\text { faire spécifiques à } \\
\text { l'utilisation du bouilleur dans } \\
\text { cet environnement }\end{array}$ \\
\hline
\end{tabular}




\begin{tabular}{|c|c|c|c|}
\hline $\begin{array}{l}\text { Mécanicien } \\
\text { centrale }\end{array}$ & $\begin{array}{l}\text { Maintenance, } \\
\text { surveillance et } \\
\text { réparation des systèmes } \\
\text { de production d'énergie }\end{array}$ & $\begin{array}{l}\text { Froid : l'eau pompée de l'océan } \\
\text { gèle sur le temps de la } \\
\text { remontée au système. D'où } \\
\text { création d'un système plus } \\
\text { complexe et prototypique } \\
\text { permettant l'autonomie sur la } \\
\text { base : le bouilleur; dispose de } \\
\text { 10mn pour remettre en route le } \\
\text { système en cas de black-out } \\
\text { avant que le système ne gèle }\end{array}$ & $\begin{array}{l}\text { Utilisation d'un système } \\
\text { inconnu } \\
\text { Gestion des pannes } \\
\text { spécifiques rapidement } \\
\text { Développement de savoir- } \\
\text { faire spécifiques à } \\
\text { l'utilisation du bouilleur dans } \\
\text { cet environnement }\end{array}$ \\
\hline Plombier & $\begin{array}{l}\text { Maintenance du } \\
\text { chauffage et de la } \\
\text { chaudière }\end{array}$ & $\begin{array}{l}\text { Isolement : matériel acheminé } \\
\text { par bateau sur la période } \\
\text { d'ouverture des glaces. } \\
\text { Matériels manquant durant } \\
\text { l'hivernage. } \\
\text { Froid : les canalisations d'eau } \\
\text { gèlent }\end{array}$ & $\begin{array}{l}\text { Développement de stratégies } \\
\text { d'occupation du temps, lutte } \\
\text { contre l'oisiveté dans le } \\
\text { travail } \\
\text { Développement de savoir- } \\
\text { faire spécifiques au } \\
\text { réchauffement des } \\
\text { canalisations d'eau et pour se } \\
\text { préserver du froid }\end{array}$ \\
\hline Chef technique & $\begin{array}{l}\text { Répartition du travail } \\
\text { entre les techniciens, } \\
\text { planifie le travail sur du } \\
\text { court et moyen terme }\end{array}$ & $\begin{array}{l}\text { Isolement : les techniciens } \\
\text { viennent en aide aux } \\
\text { scientifiques } \\
\text { Vent et froid : Tout casse } \\
\text { (froid) et s'envole (vent) } \\
\text { Incertitude : Impossibilité de } \\
\text { planifier le travail des } \\
\text { techniciens dans le temps }\end{array}$ & $\begin{array}{l}\text { Gestion de l'imprévu } \\
\text { (planification impossible) } \\
\text { Fixation de tous les éléments } \\
\text { restant à l'extérieur } \\
\text { Développement de savoir- } \\
\text { faire spécifiques à chaque } \\
\text { corps de métier technique en } \\
\text { adéquation avec le froid, pour } \\
\text { organiser le travail, pour se } \\
\text { préserver du froid }\end{array}$ \\
\hline
\end{tabular}

Tableau 2: Adaptation de l'activité professionnelle aux contraintes polaires

\section{Table 2: Adaptation of professional activity to polar conditions}

L'isolement de la base ne rend pas accessible le matériel nécessaire pour les travaux à tous moments, puisqu'il est acheminé par bateau. Par exemple, c'est ce que le plombier soulève durant son entretien. Le confinement se ressent à travers le manque de place sur la base, qui incite l'organisation à imposer un choix de machine au mécanicien de précision par exemple, qui devra travailler autrement pour concevoir les pièces. Le climat a été une contrainte fréquemment énoncée durant les entretiens, spécifique à chaque corps de métier. Les températures basses et les précipitations de neige ne permettent pas la réalisation des travaux extérieurs sans adaptation. Les vents violents imposent de tout fixer en extérieur (propos du responsable technique). De plus, ils soulèvent la neige et forment des congères, rendant inaccessibles les entrées de bâtiments. La neige s'infiltre sous les portes, les fenêtres, dans les boîtiers électriques et empêche la fermeture des espaces (propos de l'électricien et du menuisier). Enfin, une dernière contrainte a été énoncée par le chef technique, celle des incertitudes omniprésentes sur la base et ne facilitant pas l'organisation du travail à la journée.

Ces contraintes impactent fortement le travail des techniciens et impliquent l'adaptation de leurs savoir-faire de manière différenciée selon le corps de métier et les tâches à réaliser.

Les résultats soulignent quatre types d'ajustement du travail face aux contraintes polaires énoncées ci-dessus : l'isolement, le confinement, le climat, l'incertitude.

\subsection{1.- Apprendre à travailler isolé avec du matériel inhabituel et des prototypes}

L'isolement du continent ntarctique et son inaccessibilité obligent à développer une large autonomie, y compris dans la réparation du matériel qui dysfonctionne. Le matériel utilisé en Antarctique, les outils, les systèmes de production d'eau ou de chauffage, les engins à chenille, les fermetures des portes et des fenêtres des bâtiments, les systèmes électriques et les systèmes utilisés sur la base sont des prototypes répondant au mieux aux contraintes polaires. La spécificité de l'environnement polaire surprend et désarme parfois les hivernants dans leur pratique professionnelle, éloignée de leur formation initiale. Par exemple, le 
mécanicien de véhicule découvre les engins utilisés en Antarctique : "Ici les engins sont atypiques !!! Moi je suis habitué aux camionnettes, aux voitures, mais pas au muskeg...(...). Faut réchauffer le moteur et les boîtes et je savais pas...»(MV). Des techniques spécifiques ont été développées par les hivernants afin de réguler leur activité professionnelle pouvant apporter une solution aux problématiques rencontrées durant l'hivernage. Les nouvelles techniques se sont ajustées à la spécificité du matériel existant sur place, ce qui a modifié la pratique professionnelle des hivernants. Cet ajustement reste propre à chacun et provient la plupart du temps de création et d'innovation. Par exemple, le mécanicien de véhicule, face aux difficultés rencontrées pour démarrer les engins en hiver, a confectionné des systèmes de couvercles avec des plastiques pour éviter les infiltrations de neige. Ou encore, il trouve le moyen de réchauffer les moteurs en installant des tuyaux soufflant de l'air chaud sous les capots : "Il y avait de la neige dans le filtre à air donc j'ai trouvé un système de couvercle sur l'entrée d'air. Et on peut pas démarrer l'engin avec ce cache (...). Pour démarrer les engins, les liquides ne doivent pas être trop froids, donc on les réchauffe. J'ai fait une plaque pour fixer les résistances, et j'ai installé des panneaux chauffants sur les boîtes de vitesses»(MV). Les hivernants ont développé donc «de nouveaux savoir-faire» pour travailler avec des engins ou des matériaux spécifiques utilisés en contexte polaire.

\subsection{2.- Apprendre à travailler confiné avec du matériel inapproprié}

Le confinement et la place réduite de la base limitent le nombre de machines pour confectionner les pièces pour le mécanicien de précision. Il doit alors se débrouiller pour confectionner une pièce ronde avec une machine qui ne le permet pas, à l'origine : "En France tu as deux ou trois fraiseuses différentes donc t'as le choix. Ici y a qu'une fraiseuse et faut tout faire avec, donc ça c'est dur au niveau matériel » (MP). Il rencontre aussi des difficultés de conversion entre les plans fournis et le mode métrique des machines: "Les engins à chenille ne viennent pas d'Europe, ça vient du Canada, et le Canada travaille avec la normalisation américaine. Ici les machines que j'utilise sont à la norme européenne...». Le plombier évoque les problèmes de disponibilité du matériel qui n'a pas été acheminé (par bateau) : "Y a des problèmes de matériel qui n'arrive pas et de pièces manquantes donc les travaux ne sont pas possibles. Par exemple cet hiver j'ai pas pu faire les travaux de plomberie dans le bâtiment, y avait pas le matériel...» (P).

\section{1 .3.- Apprendre à se protéger et travailler dans le froid}

Travailler dans le froid nécessite un ajustement des modes opératoires utilisés par les techniciens. L'électricien avoue que les méthodes de travail sont spécifiques au contexte polaire : "Ce sont les méthodes de travail qui sont particulières, c'est dû au froid. Ici il ne faut jamais travailler les mains nues sinon on a les outils collés aux mains, ils sont froids, idem avec les vis » (E). Les conditions extrêmes induisent l'usage de vêtements permettant une isolation vestimentaire importante. Savoir se vêtir est un des premiers savoir-faire appris par les hivernants. Toutefois, se couvrir pour éviter les risques d'hypothermie induit également une adaptation de la dextérité ainsi qu'un accroissement du temps nécessaire à la réalisation de tâches effectuées en extérieur du fait du manque de sensation produit par les types de gants utilisés, par exemple. L'électricien le note lorsqu'il dit que «Le problème c'est qu'avec les gants on ne sent rien » (E). Pour le mécanicien de véhicule, les gants ne sont pas adaptés au travail extérieur: "Pour travailler, on est dehors, il fait froid et avec les gros gants on peut ne rien faire. (...) Tu mets plus de temps à faire les choses. Même au niveau de la respiration, le froid, ça bloque » (MV).

Au-delà des modes opératoires, les techniques utilisées varient du fait des conditions ou de l'usage de matériaux permettant de résister aux températures extrêmes. Le chaudronnier note que certaines techniques utilisées en France ne sont pas exploitables en Antarctique, notamment pour l'assemblage de grosses pièces entre elles: "La soudure gaz n'est pas possible, il y a trop de vent ici »(C). La spécificité du matériel utilisé en Antarctique est abordée par l'électricien : "Ici, il faut des câbles silicones, sinon ça casse »(E). Le 
responsable technique explique l'impact des températures sur le système de production d'eau : "Je me suis fait avoir sur 2-3 pts. Par exemple les canalisations d'eau qui gèlent quand on baisse les débits. Si c'est pas expliqué on sait pas et c'était écrit nulle part. On a passé 2 jours dans le froid pour dégeler et comprendre ce qui se passait»(RT).

\section{1 .4.- Apprendre à travailler dans l'incertitude}

Le contexte polaire, notamment les incertitudes liées aux conditions climatiques, affecte l'organisation du travail effectué sur la base, lorsque les travaux sont menés à l'extérieur. Le travail dans le froid implique d'une part plus de temps pour réaliser la même opération que dans des conditions thermiques plus favorables: "Par exemple, pour le travail sur les caravanes du RAID ${ }^{2}$, il faisait $-32{ }^{\circ} \mathrm{C}$. On y arrive, mais ça demande plus de préparation et de temps. Il faut déneiger, penser à réchauffer les canalisations pour éviter que ça gèle » (RT). D'autre part, elle implique également de s'adapter aux aléas climatiques qui n'ouvrent parfois que quelques fenêtres temporelles courtes pour effectuer le travail : "Le problème ici c'est le climat. On est tributaire de l'épaisseur de la glace. Les conditions sont difficiles et on peut être cloué dans les bâtiments. L'électricien complète : "Y a des spécificités au site par rapport à la neige, au froid. C'est du quotidien donc on l'écrit pas, car le froid c'est banal ici. On s'adapte».

Les opérateurs doivent gérer ces incertitudes permanentes et composer de manière la plus efficace possible pour effectuer le travail nécessaire au bon fonctionnement de la base. L'organisation de leur travail se voit sans cesse modifiée dans ces conditions. Par exemple l'électricien précise qu'il ne pourra pas ranger le câble électrique immédiatement après utilisation lors de travaux extérieurs : "Les câbles électriques sont souples jusqu'à-15 ${ }^{\circ} \mathrm{C}$ et au-delà... il faut attendre que les températures remontent pour les ré-enrouler »(E).

Le mécanicien de véhicule explique comment il a organisé son activité lorsqu'il répare les engins en panne à l'extérieur et les stratégies qu'il a développées pour faire face aux contraintes polaires : "Tu remontes tous les $1 / 4 d^{\prime} h$, car il fait trop froid et après il faut $1 / 2 h$ pour te réchauffer» $(\mathrm{MV})$.

Par conséquent, les opérateurs disposent d'une grande latitude et liberté d'action. Ainsi, les techniques utilisées, les méthodes de travail ou l'organisation du travail à faire sont peu standardisées. L'opérateur devra s'ajuster en permanence aux contraintes en temps réel.

\section{2.- Les ressources et leurs usages pour adapter le travail}

\subsection{1.- La diversité des affordances offertes}

Plusieurs ressources sont à disposition des techniciens de la base leur permettant d'adapter leur activité aux contraintes. Ces ressources dessinent un champ de possibilités variées d'usage par les opérateurs. En cela, elles sont des capabilités d'usage. Ces ressources sont :

1. les documents existants sur place, comme les Comptes Rendus d'Activités Mensuels rédigés par le prédécesseur (CRAM). Ce document officiel, instauré par l'IPEV, doit être rempli chaque mois. Il fait état des incidents rencontrés et des actions menées.

2. les notes personnelles rédigées à l'occasion de la relève de poste avec le prédécesseur. Cette relève de poste a lieu après 12 à 14 mois d'activité sur la base sans interruption.

3. les documents propres aux systèmes de la base comme les manuels d'utilisateur à disposition ;

4. une aide potentielle d'un technicien plus expérimenté sur la base ;

5. la possibilité offerte d'entrer en contact mail avec un référent de l'IPEV.

Ces ressources ne préjugent pas de l'usage qui en est fait par les opérateurs techniciens sur la

2 Convoi terrestre permettant de transporter le fuel, les denrées alimentaires et le matériel en vue de la préparation de l'hivernage à Concordia, base franco-italienne, située à $1150 \mathrm{~km}$ de DDU, à l'intérieur du continent. 
base pour s'adapter aux contraintes. La mise en évidence des choix effectués par les techniciens permet de saisir une hiérarchie implicite dans l'usage de ces ressources.

\subsection{2.- L'usage des ressources : hiérarchie implicite et dimension constructive de l'activité}

C'est la recherche personnelle d'une solution aux problèmes qu'ils rencontrent qui organise les techniciens dans l'usage des ressources potentielles. Par exemple, l'électricien explique qu'il se débrouille pour effectuer ses tâches : "je fais comme je sens, le tout c'est que ce soit fait»(E). Le mécanicien de précision aboutit au même constat lors de la conception de pièces spécifiques : "Ici faut faire un rond, même si t'as pas la machine, tu te débrouilles pour faire un rond, tu fais à l'ail, tu te débrouilles » (MP). Ces propos sont confirmés par le mécanicien de véhicules : "Je me suis débrouillé. J'ai cherché par moi-même » (MV). Quel que soit le corps de métier, les techniciens ont procédé de manière autonome pour adapter leur activité. Pour le responsable technique «à part le tri des déchets j'ai appris par moi même » (RT).

Ainsi, l'apprentissage s'effectue principalement de manière autonome en mobilisant les ressources qui les contraignent le moins. Les contraintes de l'activité productive et l'autonomie laissée par l'organisation du travail sur la base Dumont D'Urville permettent aux opérateurs de rechercher activement des solutions qu'ils ajustent au fur et à mesure «par essai-erreur ». En ce sens, les ressources utilisées par les opérateurs sont d'abord les ressources qui leur permettent de faire par eux-mêmes et de faire les choses « au feeling ».

Pour illustrer, le mécanicien de précision explique qu'il a utilisé des livres et des documents pour travailler les conversions puis il précise qu'il a appris «sur le tas », par essai erreur : "J'ai essayé de bosser les conversions avec les bouquins, j'ai appris sur le tas, on m'avait pas prévenu (...). Y a pleins de trucs que j'ai fait à l'œil ou au feeling » (MP). Le plombier a cherché dans la documentation pour trouver la panne: "Tu vas au BT (Bâtiment Technique), y a toutes les notices techniques. Et après, c'est du bon sens » $(\mathrm{P})$.

Lorsque les contraintes productives deviennent trop fortes et les marges de manœuvre temporelles pour employer ces stratégies personnelles d'usage, les opérateurs se rabattent sur des ressources qui leur permettent de sauvegarder le plus possible leur autonomie. Pour illustrer, après avoir cherché les solutions par lui-même, le mécanicien de véhicule a cherché de l'aide auprès des autres techniciens : "J'ai cherché par moi-même et pour pas perdre de temps j'ai demandé aux autres » (MV). Ils peuvent ainsi (1) faire appel à des aides d'autres techniciens présents sur la base; (2) rentrer en contact mail avec le référent de l'Institut Polaire Français, en France.

Pour résumer, les techniciens s'orientent avant tout vers des ressources leur permettant de rechercher par eux-mêmes les solutions aux problèmes qu'ils rencontrent dans leur activité productive sur la base. Les deux dernières ressources potentiellement utilisables sont celles qui leur laissent moins de liberté d'action : elles sont utilisées seulement pour pouvoir gagner du temps ou pour répondre aux contraintes productives lorsque les aléas climatiques ont réduit les marges de manœuvre temporelles pour effectuer certaines tâches.

\section{4.- Discussion}

Nous organiserons cette discussion en trois temps. Un premier temps a trait aux liens entre les contraintes polaires et la recherche d'autonomie des opérateurs dans l'organisation de leur travail. Un second temps concerne l'usage des ressources par les opérateurs et la définition d'une contrainte acceptable. Un troisième temps enfin établit une articulation entre activité productive et activité constructive. Ces trois temps permettront de discuter de l'apport des deux modèles (celui de l'environnement capacitant et celui du Workplace Learning) au regard de l'apprentissage. 


\section{1.- L'autonomie comme ressource essentielle pour apprendre et se protéger}

L'environnement polaire exerce un nombre important de contraintes sur l'activité des opérateurs. Parmi ces contraintes, les températures extrêmes et les conditions climatiques difficiles provoquent une grande incertitude quant au moment de réalisation des tâches de travail. Les techniciens sont de fait constamment soumis à des incertitudes temporelles dans la réalisation de leur travail, rendant difficile la planification du travail. L'organisation du travail laisse de ce point de vue de grandes marges de liberté aux opérateurs dans la réalisation de leurs tâches, dans la possibilité d'essayer de nouvelles manières de faire, et finalement, d'apprendre.

C'est d'ailleurs cette autonomie dans le travail, cette capacité à trouver les solutions, seuls face aux problèmes professionnels qu'ils rencontrent, que les opérateurs valorisent leur travail sur la base. Dans ces conditions extrêmes, le travail s'effectue sans prescription «descendante » (Daniellou, 2002; Six, 1999) dans le sens où les manières de faire, les modes opératoires, comme les moments de réalisation des tâches ne sont pas définis avec précision. Cette organisation spécifique laisse en définitive de grandes marges de liberté aux opérateurs et leur permet de développer des compétences à la fois dans l'organisation de leur propre travail, mais également dans les savoir-faire spécifiques que le travail requiert dans cet environnement particulier. Ainsi, cette marge de liberté est une ressource indispensable pour le développement des opérateurs : elle leur permet d'adapter leur travail en utilisant prioritairement des ressources privilégiées, c'est-à-dire celles qui leur permettent d'être autonomes.

\section{2.- Contraintes acceptables et engagement des opérateurs}

L'usage des ressources à disposition des opérateurs pour adapter leur travail aux différentes conditions est d'ailleurs significatif de cette volonté de préserver leur autonomie. Si l'apprentissage est lié à la congruence entre d'une part, les affordances et d'autre part, l'engagement des opérateurs, il est alors possible d'argumenter que l'usage des ressources par les opérateurs constitue un indicateur indirect de leur engagement dans leur travail. Les résultats montrent que les opérateurs mobilisent en premier lieu les manuels techniques, ou les notes laissées par leurs prédécesseurs comme les CRAM. On le voit ici, ce n'est pas l'appui de l'expertise de l'IPEV ou la demande d'aide à un autre technicien qui est privilégiée. Même si ce sont des ressources, elles ne sont envisagées que comme les dernières solutions pour réaliser le travail. On retrouve ici la question de l'adéquation nécessaire entre les ressources mobilisées par les opérateurs et leur engagement dans la situation, développée par Billett $(2001 ; 2012)$. Cette forme d'engagement explique la hiérarchie dans l'usage des ressources offertes par l'environnement de travail. Toutefois, a contrario de cette approche mettant en avant le rôle privilégié des interactions tutorales dans l'apprentissage au travail, les opérateurs tendent ici à favoriser les ressources permettant de résoudre par eux-mêmes les problèmes auxquels ils sont confrontés, quand bien même ces ressources sont construites sur la base de l'expérience de leurs prédécesseurs. Ainsi, malgré les conditions de travail extrêmes sur la base DDU, les opérateurs techniciens adaptent leur activité en cherchant activement des solutions aux problèmes qu'ils rencontrent. Ils ne demandent une aide que dans les cas où les contraintes temporelles ne leur laissent plus d'autres possibilités pour construire une réponse. La préservation de leur autonomie et de leur marge de liberté constitue de ce point de vue un moteur essentiel à leur engagement dans le travail pour optimiser leur apprentissage-développement. À ce stade, il est possible de définir ce qu'est une contrainte acceptable du point de vue d'un environnement capacitant : c'est une contrainte qui implique l'adaptation des opérateurs au plan de leur activité productive et qui leur permet de mobiliser les ressources de l'environnement par leur engagement en situation. Il s'agit là d'un apport important de l'approche Workplace Learning qui consiste à conceptualiser cette dimension personnelle d'engagement dans la modélisation de l'apprentissage au travail. 
Il reste cependant à revenir sur la question de contrainte permettant l'apprentissage. En ergonomie, le travail en conditions extrêmes est regardé avant tout sous l'angle des contraintes. Ainsi, dans leur article Wolff et Sperandio (2004) abordent le travail sous fortes contraintes thermiques du point de vue des risques, mais pas du point de vue de l'activité que les opérateurs déploient, ni de celui des savoirs qui permettent d'agir. Sur la base DDU, les opérateurs développent des savoir-faire de prudence pour se prémunir des conditions extrêmes. Se vêtir de manière adaptée, limiter le temps de travail en extérieur, attendre ou planifier son travail pour le réaliser dans des conditions climatiques plus favorables sont par exemple des savoir-faire qui impactent les modalités de travail des opérateurs tant sur le plan des techniques utilisées, que des modes opératoires mis-en-œuvre. Certes, l'environnement apparaît à risque, mais limité par l'autonomie de l'opérateur et les marges de manœuvre temporelles suffisantes pour travailler et apprendre de son travail. De ce point de vue, l'environnement de travail des opérateurs est extrême, mettant en œuvre des savoir-faire de prudence impliquant l'apprentissage de nouveaux savoir-faire de métier. Dans ce cadre, il nous semble que l'ergonomie constructive permet comparativement à l'approche Workplace Learning un apport intéressant : celui des relations entre les trois niveaux d'environnements (préventif, universel, développemental). Sur la base DDU, c'est la dimension préventive de l'environnement de travail (grande latitude organisationnelle et savoir-faire de prudence) qui constitue à la fois une ressource pour la protection des opérateurs et, à la fois comme une contrainte permettant l'apprentissage/développement de nouveaux savoir-faire de métier.

\section{3.- Contraintes productives, stratégies constructives et engagement des opérateurs}

La dimension productive du travail des techniciens de la base DDU est nécessaire à la survie des hivernants. Ces derniers sont donc à la recherche d'une efficacité, qui aura pour effet d'enrichir la dimension constructive du travail, en termes de développement de nouvelles compétences. Dans ce cadre, l'activité constructive est bien un effet de l'activité productive (Samurçay \& Rabardel, 2004). Toutefois, la prévalence de l'une ou l'autre de ces dimensions (constructive ou productive) est liée aux aléas et à l'incertitude des conditions polaires. Ainsi, lorsque les conditions climatiques sont favorables et laissent des marges de manœuvre temporelles, c'est la dimension constructive qui est privilégiée : les opérateurs cherchent activement des solutions aux problèmes qu'ils rencontrent, ils testent de nouvelles solutions, ils innovent et sont créatifs, font des erreurs et recommencent. En ce sens, on pourrait dire qu'ils mettent pleinement en jeu une activité propre au sens où l'entend Clot (2013) : ils s'organisent, produisent par eux-mêmes et laissent une trace dans leur environnement dans lequel ils se reconnaissent. Dans ce cadre, nous l'avons vu, l'engagement des opérateurs est central pour comprendre l'apprentissage au travail. Les marges de manœuvre temporelles laissent une latitude permettant un apprentissage effectif.

A contrario lorsque du fait des aléas climatiques les marges de manœuvre temporelles se réduisent, la dimension constructive est minorée au profit de la dimension productive : il faut réaliser le travail dans le temps imparti et mobiliser pour ce faire des ressources non privilégiées. Les techniciens font par exemple appel via le mail à un référent de l'IPEV plutôt que d'essayer de construire par eux-mêmes une solution. La dimension productive prend le pas sur la dimension constructive, et l'engagement des opérateurs (i.e. la recherche d'autonomie) est mis de côté.

Ici, les marges de manœuvre productives doivent être différenciées des marges de manœuvre constructives. Faire et apprendre à faire ne sont pas les mêmes activités et ne nécessitent pas la même temporalité. La différence se situe essentiellement dans l'usage de ressources concordantes ou non avec l'engagement des opérateurs. Du point de vue des environnements capacitants, on pourrait dire que la marge de manœuvre temporelle constitue non pas un facteur de conversion en soi, mais est seulement susceptible de l'être au regard de la prévalence productive ou constructive de l'activité des opérateurs. Par exemple, si le 
technicien qui a une marge de manœuvre réduite du fait des conditions climatiques fait appel à une ressource non concordante avec son engagement, il préserve les marges de manœuvre productives pour agir tout en se protégeant, mais limite ses capabilités d'apprendre. Si les conditions climatiques n'impactent pas les marges de manœuvre temporelles, celles-ci constituent pour les opérateurs des facteurs de conversion permettant les capabilités d'apprendre soi-même en mobilisant des ressources concordantes avec son engagement dans la situation de travail pour se construire.

\section{Conclusion}

Cet article visait à confronter deux modèles de l'apprentissage-développement (l'approche des environnements capacitants et l'approche Workplace Learning) aux résultats d'une recherche-intervention menée sur la base Antarctique Dumont D’Urville. La nécessité pour les opérateurs d'adapter leurs savoir-faire pour réaliser leur travail et pour assurer la survie des hivernants en fait un terrain d'étude privilégié pour comprendre les stratégies constructives et la mobilisation de leurs ressources pour adapter leur travail.

Dans cet environnement extrême, nous avons montré que l'autonomie et les marges de manœuvre laissées aux opérateurs constituent à la fois une ressource pour se protéger et pour apprendre. C'est cette latitude laissée aux opérateurs qui permet la mise en œuvre effective des savoir-faire de prudence et l'apprentissage-développement de savoir-faire de métiers. Les ressources mobilisées et hiérarchisées par les opérateurs mettent en avant un apport important de l'approche Workplace Learning: la conceptualisation de la notion d'engagement est fondamentale dans la mobilisation des ressources par les techniciens pour apprendre sur la base Dumont D'Urville. De manière complémentaire, l'approche d'ergonomie constructive, comparativement à celle du Workplace Learning, met l'accent sur les liens existant entre les niveaux préventifs et d'apprentissage-développement des environnements capacitants. Dans cette voie, c'est au regard de l'activité des opérateurs, et de leur engagement en situation qu'il faut comprendre les concepts avancés par l'ergonomie constructive à la suite des travaux de Sen. La notion de facteur de conversion ne peut-être comprise qu'en référence à l'activité des opérateurs dont la dimension prédominante est soit productive, soit constructive, selon les marges de manœuvre temporelles laissées par les conditions climatiques.

À ce titre, nous pouvons maintenant revenir sur la définition des environnements capacitants pour en proposer une définition qui ne tienne pas seulement compte des niveaux d'action de l'ergonome (Falzon \& Mollo, 2009), mais qui inclut également la notion d'engagement issue de l'approche Workplace Learning. Ainsi, sur la base des résultats de cette étude, nous avançons qu'un environnement de travail qui favorise le développement et l'apprentissage dans et par le travail :

— offre des contraintes acceptables du point de vue de l'apprentissage-développement. Ces contraintes sont acceptables si elles sollicitent et impliquent l'adaptation de l'activité productive, et si elles peuvent être surmontées grâce à l'usage de ressources concordantes avec l'engagement des opérateurs dans les situations de travail.

— laisse des marges de liberté et des marges temporelles suffisantes dans la réalisation du travail, autorisant l'erreur. Ce sont ces marges de manœuvre qui orientent les opérateurs vers l'emploi de stratégies innovantes et l'affinement progressif des solutions trouvées. Dans ce cas, l'activité constructive relaie l'activité productive.

- engage pleinement les opérateurs dans une activité propre, au sens où ils peuvent agir sur leur environnement, s'organiser dans leur travail et laisser dans l'environnement une trace dans laquelle ils peuvent se reconnaître. 


\section{BIBLIOGRAPHIE}

Billett, S. (1992). Developing vocational skills in the workplace. Australian Journal of TAFE Research and Development, 8(1), 1-12

Billett, S. (2001). Learning through work: workplace affordances and individual engagement. Journal of workplace Learning, 13(5), 209-214.

Billett, S. (2002). Workplace pedagogic practices: Co-participation and learning. British Journal of Educational Studies, 50(4), 457-481.

Billett, S. (2009). Conceptualizing learning experiences: Contributions and mediations of the social, personal and brute. Mind, Culture and Activity, 16(1), 32-47.

Billett, S., Barker, M., \& Hernon-Tinning, M. (2004). Participatory practices at work. Pedagogy, Culture \& Society, 12(2), 233-258.

Billett, S., Johnson, G., Thomas, S., Sim, C., Hay, S \& Ryan, J (Eds.) (2012) Experiences of school transitions: Policies, practice and participants. Dordrecht, The Netherlands: Springer.

Bourgeois, E. \& Durand, M. (2012). Apprendre au travail. Paris: PUF.

Clot, Y. (2013). Développer le métier. Le collectif dans l'individu. Actes de la conférence inaugurale des 7es biennales de l'ARIS. Amiens: Presses Universitaires d'Amiens.

Corbin, J., \& Strauss, A. (2008). Basics of qualitative research: Techniques and procedures for developing grounded theory (3rd ed.). Thousand Oaks, CA: Sage.

Cru, D. (1995). Règles de métier, langue de métier : dimension symbolique au travail et démarche participative de prévention. Le cas du batiment et des travaux publics. Mémoire, EPHE, Paris.

Daniellou, F. (2002). Le travail des prescriptions. Conférence inaugurale au XXXVIème congrès de la SELF, Aix-en-Provence.

Delgoulet, C., \& Vidal-Gomel, C. (2013). Le développement des compétences : une condition pour la construction de la santé et de la performance au travail. In P. Falzon (Ed.), Ergonomie constructive (pp. 19-32). Paris: PUF.

Falzon, P. (2005). Ergonomics, knowledge development and the design of enabling environments, Humanizing work and wok environments (pp.1-8). Humanizing Work and Work Environment HXXE'2005 Conference, Guwahati, India.

Falzon, P. (2006). Enabling safety: issue in design and continuous design. In design process and human factors integration: optimizing company performance (pp. 1-12). $9^{\text {th }}$ ISSA International Symposium, Nice, France.

Falzon, P. (2013). Ergonomie constructive. Paris: PUF.

Falzon, P., \& Mollo, V. (2009). Pour une ergonomie constructive : les conditions d'un travail capacitant, Laboreal, 5(1), 61-69.

Gibson, J.J. (1977). The theory of affordances. In R. Shaw \& J. Bransford (Eds.), Perceiving, Acting and Knowing (pp.67-82). Hillsdale, NJ: Erlbaum.

Glassman, M. (2001). Dewey and Vygotsky: society, experience and inquiry in educational practice. Educational Researcher. Educational Researcher, 30(4), 3-14.

Grison, B.,\& Riff, J. (2002). Validité écologique et situations d'étude privilégiées : de la psychologie expérimentale à l'anthropologie cognitive située. In Actes 4èmes Journées d'Étude de l'Association ACT'ING, "Objets théoriques, objets de conception, objets d'analyse et situations d'étude privilégiées », 6-7 juin, Domaine de Chalès, Sologne

Pavageau, P., Nascimento, A., Falzon, P. (2007). Les risques d'exclusion dans un contexte de transformation organisationnelle. Pistes, 9(2), 1-17.

Rabardel, P. (1995). Les hommes et les technologies. Approche cognitive des instruments

Contemporains. Paris: Armand Colin.

Samurçay, R., \& Rabardel, P. (2004). Modèles pour l'analyse de l'activité et des compétences : propositions. In R. Samurçay, \& P. Pastré (Eds.), Recherches en didactique professionnelle (pp. 163-180). Toulouse: Octarès.

Sen, A. (1993). Éthique et économie et autres essais. Paris: PUF.

Sen, A. (2000). Un nouveau modèle économique. Développement, justice, liberté, Paris: Odile Jacob.

Sen, A. (2012). L'idée de justice. Paris: Flammarion. 
Six, F. (1999). De la prescription à la préparation du travail : apport de l'ergonomie à la prescription et à l'organisation du travail sur les chantiers de bâtiment. Document pour l'habilitation à diriger les recherches, Université Charles de Gaulle, Lille 3.

Vygotski L. (1934/1997). Pensée et langage. Paris: La Dispute

Wolff, M. \& Sperandio, J.-C. (2004). Le travail en conditions extrêmes. In P. Falzon (Ed.), Ergonomie (pp. 115-128). Paris: PUF.

Zimmerman, B. (2008). La liberté au prisme des capacités. Paris: Éditions EHESS.

\section{RESUME}

L'apprentissage sur le lieu de travail est devenu un réel enjeu de recherche. Dans ce cadre, l'ergonomie constructive (Falzon, 2013) propose le concept d'environnement capacitant pour modéliser les caractéristiques d'un environnement permettant l'apprentissage et le développement dans et par le travail. L'objectif de l'article est d'étudier l'apprentissage-développement d'opérateurs hivernants, en mettant en tension deux modèles pour éclairer les conditions favorisant l'apprentissage au travail: l'approche des environnements capacitants et celle du Workplace Learning développé par Billett (2001). L'article propose une étude du travail de neuf techniciens hivernants, isolés pendant douze mois sur la base française en Antarctique, à Dumont D'Urville. Les résultats montrent que les contraintes polaires, l'organisation du travail qui laisse de l'autonomie aux opérateurs, ainsi que l'usage spécifique des ressources disponibles permettent le développement de nouveaux savoir-faire. En ce sens, l'article discute et avance une définition des caractéristiques des environnements permettant l'apprentissage dans et par le travail.

\section{MOTS-CLES}

Apprentissage, activité productive, environnement capacitant, conditions polaires, engagement

\section{RÉFÉRENCEMENT}

Villemain, A., \& Lémonie, Y. (2014). Environnement capacitant et engagement des opérateurs : une mise en débat à partir de l'activité des techniciens de la base polaire Dumont d'Urville. Activités, 11(2), 26-43. http://www .activites.org/v11n2/v11n2.pdf

Article soumis le 17 juillet 2013, accepté pour publication le 9 juin 2014 